\title{
Linearization of Double Balanced Mixer Operated in Subthreshold Region of MOSFET
}

\author{
Sonika Gupta ${ }^{1}$, G. M. Rather ${ }^{1}$, Hakim Najeeb-ud-din (SMIEEE) ${ }^{* 1}$
}

Accepted 15 ${ }^{\text {th }}$ August 2014

Abstract: A mixer is an essential part of a communication system. It is generally used in wireless receiver to perform the translation from Radio Frequency (RF) to the intermediate frequency (IF). A non-linear device like Metal Oxide Semiconductor Field Effect Transistor (MOSFET) can be suitably used for mixing operation. In these devices, power consumption is a matter of concern because devices are continuously being scaled, but the power supply is not scaled correspondingly. In portable devices, it is important to save battery lifetime. So, it is required that the performance of mixers should be enhanced while considering power concerns also. When MOSFETs are operated in subthreshold region, there is lower power dissipation, and it has also been observed that in this region, drain current is exponential of input voltage. This nonlinearity can be used for mixing. But, this non-linearity should not be so large so as to cause distortion at the output of mixer. The distortion behavior of mixer is a matter of concern. This paper describes the results of applying linearization techniques to double balanced mixer while operating it in subthreshold region. $0.5 \mu \mathrm{m}$ NMOS devices have been used to design mixer. Mixer has been designed for Bluetooth specifications.

Keywords: Degeneration, Linearity, IIP3, Mixer, Noise Figure, Subthreshold, Third-order transconductance.

\section{Introduction}

Mixer can be designed by using nonlinear devices like diodes, BJT and MOSFETs. Each one of them has certain merits and demerits. The choice of selection depends on application and performance specifications. The nonlinearity required for mixing operation should be small. Different communication standards have emerged and they occupy narrow frequency bands in a geographic region. So, the issue of linearity is stringent in RF systems [1]. Several RF circuit issues like harmonic generation, intermodulation distortion and desensitization can be minimized by a good linearity performance. The linearity of any typical transceiver is dominated by mixers. In receiver chain, mixers are placed after the low noise amplifier (LNA). This implies that mixers have to handle amplified outputs of LNA. So, the issue of linearity becomes significant in mixers [2]. However, a trade-off exists between linearity and power consumption [3]. Lower power consumption is important due to the growth of battery-operated portable communication devices [4]. One of the approach for low power design is to use the MOSFET in the subthreshold regime of operation. When gate to source voltage $\left(\mathrm{V}_{\mathrm{GS}}\right)$ is below threshold voltage $\left(\mathrm{V}_{\mathrm{TH}}\right)$, the region of operation is called subthreshold or weak inversion.

The work in this paper is motivated by the low power consumption of MOSFET in subthreshold region of operation. Transfer function of MOSFET in subthreshold operation is nonlinear in nature, and the advantage of that has been taken in the design of mixer, as mixing is also a nonlinear operation. But this nonlinearity should not be very large to cause degradation in performance parameters. Thus, it is necessary to linearize the mixer. In this work, different linearization techniques have been

${ }^{1}$ Electronic and Communication Engineering Department, National Institute of Technology Srinagar, J \& K 190006 India

* Corresponding Author: Email: najeeb@nitsri.net

\#Note: This paper has been presented at the International Conference on Advanced Technology\&Sciences (ICAT'14) held in Antalya (Turkey), August 12-15, 2014.

This journal is (C) Advanced Technology \& Science applied to a double balanced mixer topology operating in subthreshold region.

Section II describes the necessity of linearity in systems. Linearity characterization is discussed in section III. Linearization techniques are presented in section IV. Simulation results are discussed in section $\mathrm{V}$ and concluded in section VI.

\section{Need of Linearization}

To understand the linearity requirement, consider the input-output relationship of a non-linear system, given typically as [5]:

$y=A_{1} x+A_{2} x^{2}+A_{3} x^{3}+\ldots$

Where $A_{1}$ is the ideal gain, $A_{2}, A_{3} \ldots$ are the coefficients of nonlinearity, $y$ is output signal and $x$ is input signal Consider non-linearity up to $3^{\text {rd }}$ order only. With a single tone input, say $x=A \cos w t$, output is given as:

$$
\begin{aligned}
y=\frac{A_{2} A^{2}}{2}+\left(A_{1} A\right. & \left.+\frac{3 \mathrm{~A}_{3} \mathrm{~A}^{3}}{4}\right) \cos \mathrm{wt}+\frac{\mathrm{A}_{2} \mathrm{~A}^{2}}{2} \cos 2 \mathrm{wt} \\
& +\frac{\mathrm{A}_{3} \mathrm{~A}^{3}}{4} \cos 3 \mathrm{wt}
\end{aligned}
$$

Following effects occur due to non-linearity.

\subsection{Harmonic distortion}

It is the ratio of the amplitude of a harmonic to that of the fundamental. For example, the ratio of amplitude of the tone at $3 w$ to that of the fundamental tone at $w$ is called third-order harmonic distortion $\left(H D_{3}\right)$. Applying it to equation (2):

$$
H D_{3}=\frac{A_{3} A^{2}}{4 A_{1}} \text { while assuming } \frac{3 \mathrm{~A}_{3} \mathrm{~A}^{3}}{4}
$$$$
\ll A_{1} A
$$ 


\subsection{Gain Compression}

Ideally, output power should increase with the increase in the input power. But at higher input levels, output power does not increase with input power as much as should ideally be. When the output does not increase in proportion to the input, we say gain compression is there. The concept of $1-\mathrm{dB}$ compression point is used to analyze gain compression. In equation (2), there are two terms with frequency $w$ due to non-linear behaviour. Let us assume that the other terms in $y$ have frequencies outside the band of interest and hence are removed by the band pass filter used at the output of the system. Then $y$ becomes

$$
\begin{aligned}
y=\left(A_{1} A+\frac{3 A_{3} A^{3}}{4}\right) & \cos w t \\
& =\left(A_{1}\right. \\
& \left.+\frac{3 A_{3} A^{2}}{4}\right) A \cos w t
\end{aligned}
$$

When $A_{3}$ is negative, then the second term decreases the gain.

\subsection{Blocking (Desensitization)}

Let a weak desired signal along with a strong interferer occur at the input as:

$x=a_{1} \cos w_{1} t+a_{2} \cos w_{2} t$

Where $a_{1}$ is a strong interferer and $a_{2}$ is the desired signal Applying this to equation (1), the output terms of interest (at the fundamental frequency) are:

$y=\left(A_{1} a_{2}+\frac{3 A_{3} a_{2}^{3}}{4}+\frac{3 A_{3} a_{2} a_{1}^{2}}{2}\right) \cos w t$ $+\cdots$

If the interferer strength is much greater than the desired signal strength (i.e. $\mathrm{a}_{1}>>\mathrm{a}_{2}$ ), we have:

$y=\left(A_{1}+\frac{3 A_{3} a_{1}^{2}}{2}\right) a_{2} \cos w t+\cdots$

When $A_{3}$ is negative, the small signal gain is attenuated by the interferer. If the attenuation becomes so large that the overall gain drops to zero, we say that the signal is blocked.

\subsection{Degradation of signal to noise ratio (SNR)}

Due to gain compression and blocking, the desired signal amplitude is reduced which results in degradation of SNR. In other words, due to non-linearity, frequencies outside the desired frequency are obtained at output which interfere with the desired one, behaves like noise and SNR is degraded.

\section{Characterization of Linearity}

Third order nonlinearity components are very close to fundamental frequencies and typically have highest amplitude among intermodulation products. Therefore, it is a common practice to measure nonlinearity in transmitters and receivers only up to third order [6]. A typical method for characterizing linearity is third order intercept point (IIP3). It is measured by applying two closely spaced frequencies at the input, say $f_{1}$ and $f_{2}$. Due to third order nonlinearity, different frequencies are produced at the output. These are called third order inter-modulation products. But, the most common frequencies of interest are $2 f_{1}-f_{2}$ and $2 f_{2}-f_{1}$ because they fall in the desired band and interfere with the desired frequencies. IIP3 relates third order nonlinear products to the linearly amplified and mixed signal. It is a theoretical point at the intersection of fundamental and third order curves.

\section{Linearization Techniques}

Techniques for linearizing mixers have been developed and are described as follows:

\subsection{Source Degeneration}

Source degeneration can be achieved by resistance, inductance or MOSFET. To obtain linearity, a degeneration resistor $Z_{S}$ is placed in series with the source terminal as shown in Fig 1. Resistive degeneration presents trade-offs between linearity, noise, power dissipation, and gain [7]. The value of resistor is chosen to achieve trade-off between dynamic range \& noise. In comparison to resistive degeneration, inductive degeneration is preferred due to its low noise. The Noise Figure (NF) generated in the circuit due to inductive degeneration is same as that would have been produced without degeneration i.e. noise figure is less [8]. Moreover, the circuit is more linear with inductive degeneration in comparison to resistive degeneration. The size of the circuit increases by inductive degeneration.

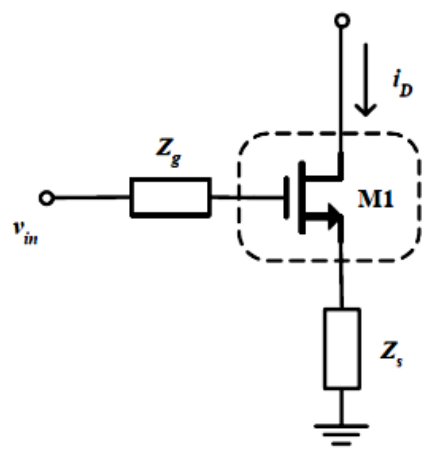

Figure 1 Source Degeneration [9]

For increasing linearity further, a capacitor can be added in parallel to gate to source capacitance.

MOSFET can be used as a resistor when biased in deep triode region. In this region, MOST is more linear than in saturation region. So, instead of using a resistor for degeneration, MOSFET can be used. But, it is affected by process and temperature variations which may degrade linearity [10].

\subsection{Third Order Transconductance Cancellation}

For a common-source MOSFET, the drain current can be given by [2]:

$i_{D S}=I_{D C}+g_{m} v_{g s}+g_{m 2} v_{g s}^{2}+g_{m 3} v_{g s}^{3}+\cdots$

Where $v_{g s}$ and $g_{m(n)}$ represents the gate to source voltage and nth - order transconductance respectively. The third order transconductance $g_{m 3}$ performs an important role in nonlinearity. The IIP3 can be increased by decreasing it.

Third order transconductance cancellation technique is used in different circuits to increase linearity [11] [12]. In this technique, the cancellation of $g_{m 3}$ is achieved by a negative peak $g_{m 3}$ transistor combined in parallel with a positive peak $g_{m 3}$ transistor. The characteristics of $g_{m 3}$ can be adjusted by adjusting the threshold voltage $\left(\mathrm{V}_{\mathrm{TH}}\right)$ of auxiliary transistor. This can be done by using body effect technique. Generally, a negative bulk to source voltage $\left(\mathrm{V}_{\mathrm{BS}}\right)$ is applied to auxiliary transistor and its $\mathrm{V}_{\mathrm{TH}}$ is increased. Size of the two transistors are chosen in such a way that 
for the same input voltage to main and auxiliary transistor, auxiliary transistor operates in weak inversion region and main transistor in strong inversion region; and a flat $g_{m 3}$ region is obtained by combining these two transistors in parallel. The compensation results are shown in Fig 2 for main transistor operating in weak inversion and auxiliary transistor operating in strong inversion region; with positive $\mathrm{V}_{\text {BS. The }}$ Thesults were obtained by simulations in device simulator. There is approximately $86 \%$ increase in linearity in the flat region.

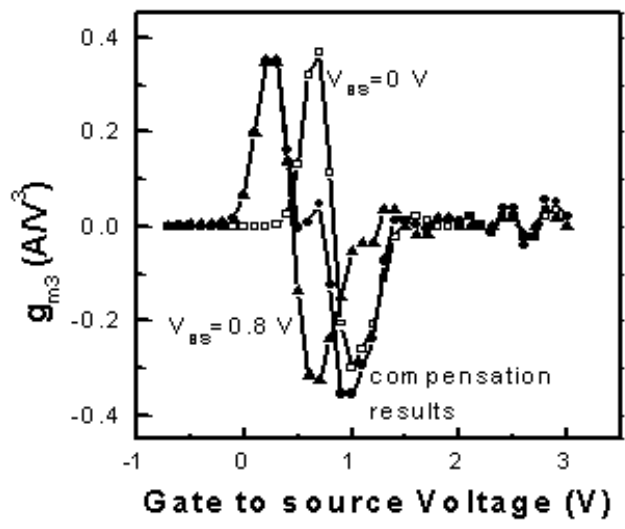

Figure 2: Compensation results of two parallel MOS Transistors This increase is only at device level. At circuit level, there will be different performance improvement.

This method does not require additional power consumption Because the total gate width of the paralleled pair can be kept equal to the actual device size of transconductance stage [2].

\section{Simulation Results and Discussions}
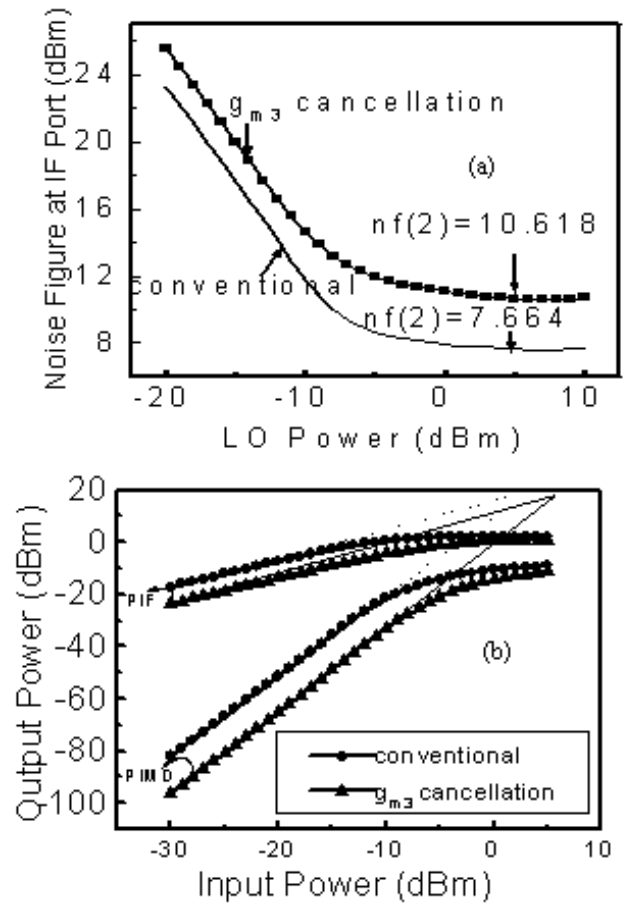

Figure 3: gm3 cancellation-Noise Figure (a) and IIP3 (b)

For simulations, specifications of Bluetooth are used i.e. radio frequency $[\mathrm{RF}]$ and Local oscillator frequency [LO] are $2.50 \mathrm{GHz}$ and $2.25 \mathrm{GHz}$ respectively [13]. $0.5 \mu \mathrm{m}$ NMOS device models were used in simulations. In all the results presented here, conventional mixer is the double balanced mixer operating in subthreshold region. Different linearization techniques were applied to this mixer. Simulations were carried in a circuit simulator. The results are presented as follows.

\subsection{Third order transconductance $\left(g_{m 3}\right)$ cancellation}

All the results are taken for mixer operating in weak inversion region; however, auxiliary transistors are biased in strong inversion region by applying positive bulk to source voltage. Simulations were carried to find optimum widths and $V_{B S}$ for maximum achievable IIP3 and without substantial degradation in gain. The NF obtained at $5 \mathrm{dBm}$ LO power is more in this technique in comparison to the conventional one as shown in Fig 3 (a). This is because more devices are added in this technique in comparison to the conventional mixer. Fig 3 (b) shows plot for finding IIP3. In the figure, PIF represents output power at one input frequency when two tones are fed at the input while PIMD represents third order inter-modulation tone due to these frequencies. IIP3 obtained in this technique is $6.138 \mathrm{dBm}$ while that in conventional mixer is $2.352 \mathrm{dBm}$. This means linearity has increased by approximately $4 \mathrm{~dB}$ by using this technique.

\subsection{Degeneration}

It is important to get optimum values of resistance, inductance and width of transistor for resistive, inductive and MOS degeneration respectively. The NF for optimum values for the three different types of degenerations is shown in Fig 4. In all the graphical plots, NFs are more than in conventional mixer. Inductive degeneration has less NF in comparison to other types as discussed previously. The IIP3 plots for the degeneration technique are shown in Fig 5. The IIP3 values obtained for resistive, inductive and MOST degeneration are $5.028 \mathrm{dBm}, 6.464 \mathrm{dBm}$ and $6.627 \mathrm{dBm}$ respectively. This implies that linearity has substantially increased. The IIP3 of mixer with inductive and MOST degeneration are comparable.

\subsection{Improved Inductive Degeneration}

In this technique, a capacitor is shunted across gate to source of inductively degenerated MOSFET in the mixer. It is necessary to select proper capacitor and inductor values, and was $0.5 \mathrm{pF}$ and 3 $\mathrm{nH}$ respectively. NF plot is shown in Fig 6 (a) where it is analysed that the NF is lesser in comparison to the conventional technique. It is also lesser than inductive degeneration NF. IIP3 plot is shown in Fig 6 (b). The value of IIP3 obtained in this technique is 7.117 $\mathrm{dBm}$, a considerable improvement.

\subsection{Combination of MOS Degeneration and $\mathrm{g}_{\mathrm{m} 3}$ Cancellation}

In this technique, MOS degeneration and $\mathrm{g}_{\mathrm{m} 3}$ Cancellation are combined and effect on performance parameters is seen. Simulations are done to find optimum voltages at gates of RF stage and LO stage while keeping other conditions same as are in individual techniques. The NF plot is shown in Fig 7 (a). It can be observed that noise figure is degraded in this technique in comparison to the conventional one and individual techniques. IIP3 plot is shown in Fig 7 (b). The value of IIP3 is $5.447 \mathrm{dBm}$ which implies that linearity is improved in comparison to conventional mixer. But it is degraded in comparison to individual techniques. 

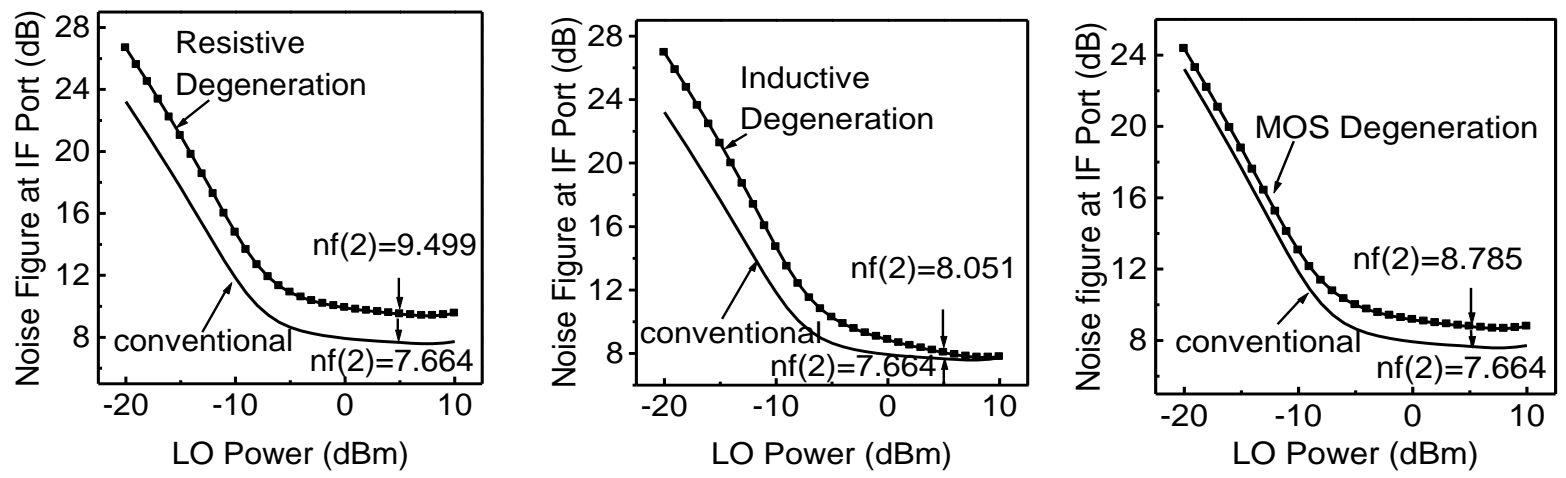

Figure 4: Resistive, Inductive and MOS Degeneration-Noise Figure
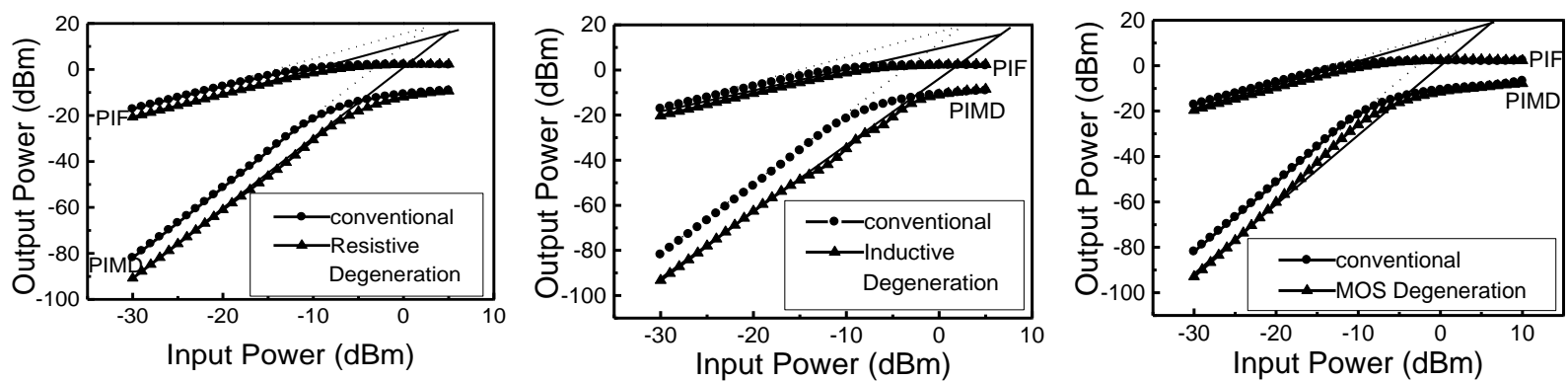

Figure 5: Resistive, Inductive and MOS Degeneration-IIP3
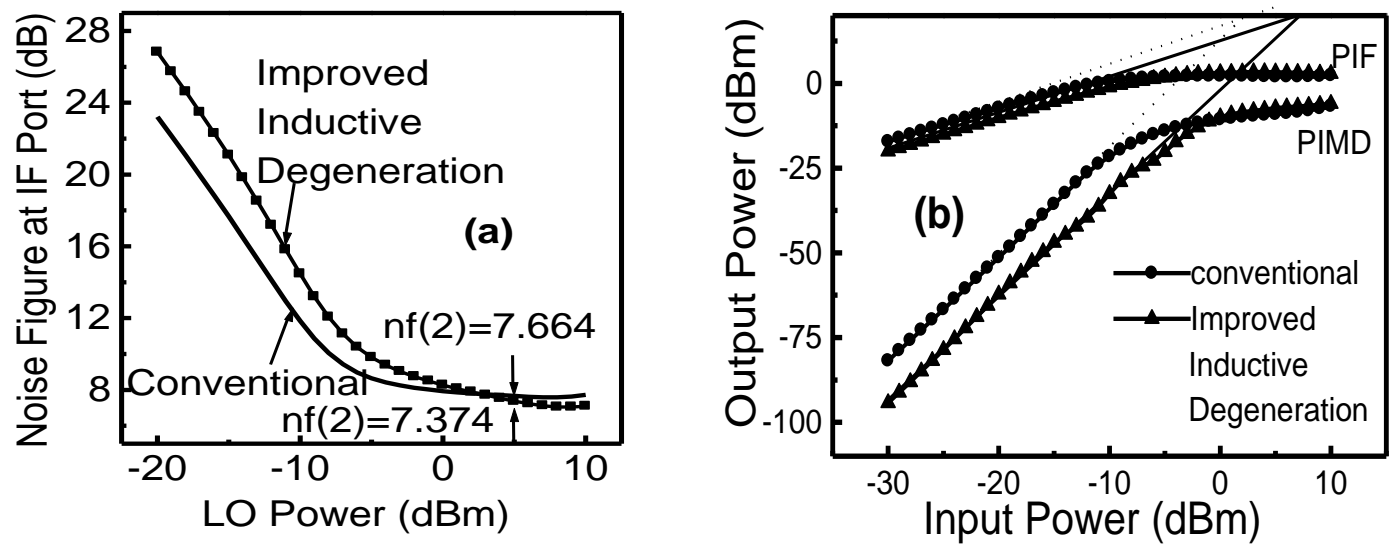

Figure 6: Improved Inductive Degeneration-Noise Figure (a) and IIP3 (b)

Figure7: Combination of MOS Degeneration and Cancellation-Noise Figure (a) and IIP3 (b)
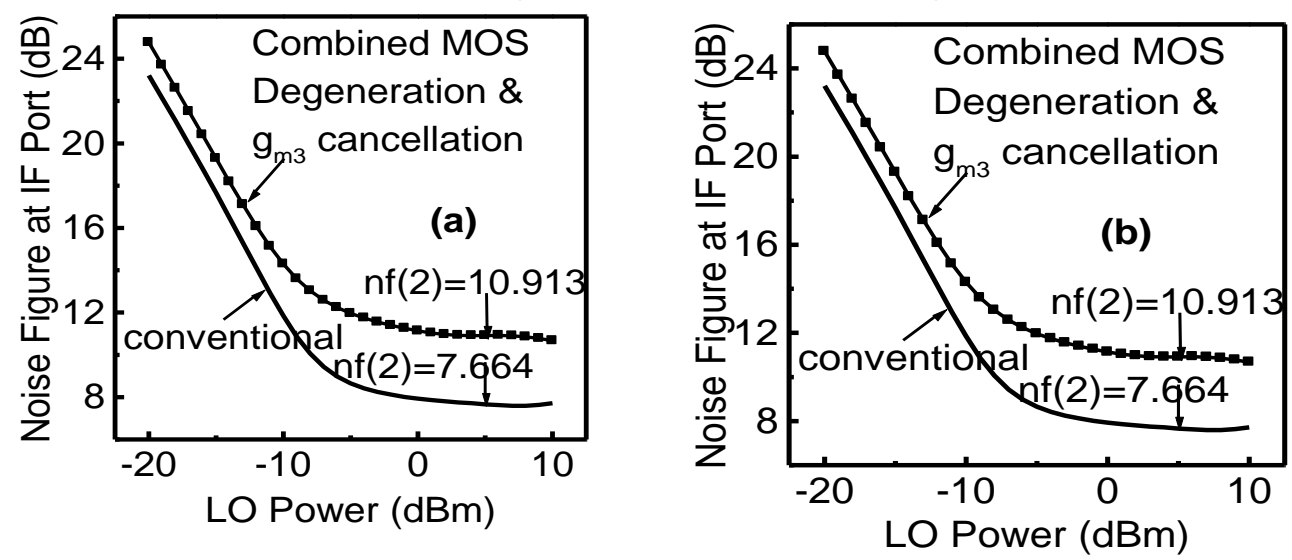

\section{Conclusion}

In this paper, we have described linearization techniques for mixers. Simulation results have been shown for applying these techniques in double balanced mixer. All the results are obtained 
for a mixer operating in subthreshold, as device consumes very low power. The results of linearization techniques applied to double balanced mixer are summarized in table 1. Figure of merit (FOM) is very good for improved inductive degeneration. It is defined as:

$$
F O M=\frac{I I P 3}{\text { Noise Figure }}
$$

Therefore, improved inductive degeneration is optimum linearization technique. There is approximately $5 \mathrm{~dB}$ increase in IIP3 in this technique and value of the noise figure is also less in comparison to other techniques. Combined MOS Degeneration and $g_{m} 3$ cancellation results are not encouraging in comparison to the individual techniques.

Table 1: Comparison of Linearization Techniques

\begin{tabular}{|c|c|c|c|}
\hline $\begin{array}{c}\text { Linearization } \\
\text { Technique }\end{array}$ & $\begin{array}{c}\text { Noise } \\
\text { Figure }(\mathrm{dB})\end{array}$ & $\begin{array}{c}\text { IIP3 } \\
(\mathrm{dBm})\end{array}$ & FOM \\
\hline $\mathrm{g}_{\mathrm{m} 3}$ Cancellation & 10.618 & 6.138 & 0.578 \\
\hline $\begin{array}{c}\text { Resistive } \\
\text { Degeneration }\end{array}$ & 9.499 & 5.028 & 0.529 \\
\hline $\begin{array}{c}\text { Inductive } \\
\text { Degeneration }\end{array}$ & 8.051 & 6.464 & 0.802 \\
\hline MOS Degeneration & 8.785 & 6.627 & 0.754 \\
\hline $\begin{array}{c}\text { Improved Inductive } \\
\text { Degeneration }\end{array}$ & $\mathbf{7 . 3 7 4}$ & $\mathbf{7 . 1 1 7}$ & $\mathbf{0 . 9 6 5}$ \\
\hline $\begin{array}{c}\text { Combined MOS } \\
\text { Degeneration } \\
\text { andg } 3 \text { cancellation }\end{array}$ & 10.913 & 5.447 & 0.499 \\
\hline
\end{tabular}

\section{Acknowledgements}

This work has been carried out in SMDP-II VLSI laboratory of the Electronics and Communication Engineering Department, of National Institute of Technology Srinagar, India. This SMDP - II VLSI project is funded by Ministry of Communication and Information Technology, Government of India. Authors are grateful to the Ministry for the facilities provided under this project.

\section{References}

[1] Heng Zhang and Edgar Sánchez-Sinencio, "Linearization
Techniques for CMOS Low Noise Amplifiers: A Tutorial," IEEE Transactions on Circuits and Systems-I: Regular Papers, vol. 58, no. 1, January 2011, pp. 22-36.

[2] Kung-Hao Liang et al., "A New Linearization Technique for CMOS RF Mixer Using Third-Order Transconductance Cancellation," IEEE Microwave and Wireless Components Letters, vol. 18, no. 5, May 2008.

[3] R. van Langevelde et al., "RF-Distortion in Deep-Submicron CMOS Technologies," IEEE Electron Devices Meeting, IEDM, Technical Digest, International, 2000, pp. 807-810.

[4] Ramesh Vaddi, S. Dasgupta, and R P. Agarwal, "Device and Circuit Design Challenges in the Digital Subthreshold Region for Ultralow-Power Applications," VLSI Design Journal Hindawi Publishing, Article No. 1, Volume 2009.

[5] Bosco Leung, VLSI for Wireless Communication, Prentice Hall Electronics and VLSI Series, 2006.

[6] Lloyd Butler VK5BR, "Intermodulation Performance and Measurement of Intermodulation Components," Available online at users.tpg.com.au/users/ldbutler/ Intermodulation.htm.

[7] Behzad Razavi, Design of Analog CMOS Integrated Circuits, Tata McGraw-Hill, 2002.

[8] Luca Daniel and Manolis Terrovitis, "A Broadband LowNoise-Amplifier," Available [online] at www.mit. edu/ dluca/.../daniel_proj_1999_microwave_lna.pdf.

[9] Moon-Su Yang et al., "Linearity Improvement Technique for the Common-Source Transconductance Stage," Available [online] at citeseerx.ist.psu.edu /viewdoc/download?doi=10.1.1.98.530.....

[10] Ko-Chi Kuo, Advances in Solid State Circuits Technologies, pp. 446, Intech Technologies, Croatia.

[11] B. Kim, J.-S. Ko, and K. Lee, "A new linearization technique for MOSFET RF amplifier using multiple gated transistors," IEEE Microwave and Guided Wave Letters, Vol. 10, No. 9, September 2000, pp. 371-373.

[12] Yen-Liang Yeh, and Hong-Yeh Chang, "Linearity Enhancement of CMOS Device using a Modified ThirdOrder Transconductance Cancellation Technique for Microwave Amplifier," IEEE MTT-S International Microwave Symposium Digest (MTT), 5-10 June, 2011.

[13] J P Silver, "Gilbert Cell Mixer Design Tutorial," Available [online] at www.scribd.com/hainals18/d/ 37009314-MOSGilbert-Cell-Mixer 